\title{
The Future of Nuclear Medicine, Molecular Imaging, and Theranostics
}

\author{
Wolfgang A. Weber ${ }^{1}$, Johannes Czernin ${ }^{2}$, Carolyn J. Anderson ${ }^{3}$, Ramsey D. Badawi ${ }^{4}$, Henryk Barthel ${ }^{5}$, Frank Bengel $^{6}$, \\ Lisa Bodei ${ }^{7}$, Irène Buvat ${ }^{8}$, Marcelo DiCarli ${ }^{9}$, Michael M. Graham ${ }^{10}$, Jan Grimm ${ }^{7,11}$, Ken Herrmann ${ }^{12}$, Lale Kostakoglu ${ }^{13}$, \\ Jason S. Lewis ${ }^{7,11}$, David A. Mankoff ${ }^{14}$, Todd E. Peterson ${ }^{15}$, Heinrich Schelbert ${ }^{2}$, Heiko Schöder ${ }^{7}$, Barry A. Siegel ${ }^{16}$, \\ and H. William Strauss ${ }^{7}$
}

${ }^{I}$ Technical University Munich, Munich, Germany; ${ }^{2}$ UCLA, Los Angeles, California; ${ }^{3}$ University of Missouri, Columbia, Missouri; ${ }^{4}$ University of California, Davis, California; ${ }^{5}$ University of Leipzig, Leipzig, Germany; ${ }^{6}$ Medizinische Hochschule Hannover, Hannover, Germany; ${ }^{7}$ Memorial Sloan Kettering Cancer Center, New York, New York; ${ }^{8}$ Institut Curie, Université PSL, Inserm, Orsay, France; ${ }^{9}$ Harvard Medical School, Boston, Massachusetts; ${ }^{10}$ University of Iowa, Iowa City, Iowa; ${ }^{11}$ Weill Cornell Medical College, New York, New York; ${ }^{12}$ University Hospital Essen, Essen, Germany; ${ }^{13}$ University of Virginia, Charlottesville, Virginia; ${ }^{14}$ University of Pennsylvania, Philadelphia, Pennsylvania; ${ }^{15}$ Vanderbilt University Medical Center, Nashville, Tennessee; and ${ }^{16}$ Washington University, Saint Louis, Missouri

\section{INTRODUCTION}

\section{Wolfgang A. Weber and Johannes Czernin}

Scientific discoveries published in The Journal of Nuclear Medicine over the past 60 years have shaped the practice of medicine and form the foundation for the future of nuclear medicine, molecular imaging, and theranostics. Nuclear medicine now provides diagnostic, prognostic, predictive, and intermediate endpoint biomarkers in oncology, cardiology, neurology, and infectious and inflammatory disorders. Whole-body target expression can be quantified and used for predicting therapy response. Treatment-induced metabolic changes serve as early prognosticators of therapy effectiveness. At the same time, technologic advances such as total-body and hybrid PET/MR imaging are revolutionizing the diagnostic capabilities of PET systems. Artificial intelligence (AI), machine learning, and radiomics have become trendy buzzwords, but their true value for clinical decision making has not yet been exploited.

However, nuclear medicine includes not only diagnostic but also therapeutic applications of radioisotopes. In fact, the successes in treating benign and malignant thyroid disorders with radioiodine were the main reasons for establishing nuclear medicine as an independent medical discipline. Radioiodine therapy also established the principle of theranostics, that is, the use of a target for both radionuclide imaging and therapy. The theranostic principle was later successfully applied to neuroblastoma, lymphomas, neuroendocrine tumors, paraganglioma, and, more recently, prostate cancer.

In this article, we the editors of The Journal of Nuclear Medicine provide an overview of what we consider the most promising future applications of nuclear medicine. We decided to focus on oncology, neurology, and cardiology as the clinically most relevant fields, and on radiopharmaceutical chemistry, instrumentation, and AI for extracting relevant image information. We hope to demonstrate that

Received Jul. 29, 2020; accepted Jul. 29, 2020.

For correspondence or reprints contact: Wolfgang A. Weber, Technical University Munich, Ismaningerstrasse 22, Munich 81675, Germany.

E-mail: w.weber@tum.de

COPYRIGHT (c) 2020 by the Society of Nuclear Medicine and Molecular Imaging. DOI: 10.2967/jnumed.120.254532 the field not only is highly relevant for diagnosing and understanding the pathophysiology of human diseases but also is increasingly becoming an integral part of patient management.

\section{ONCOLOGY}

\section{Wolfgang A. Weber, Lale Kostakoglu, Heiko Schöder, and Barry A. Siegel}

In 2020, more than 1.8 million individuals will be diagnosed with invasive cancer in the United States. Although the incidence of invasive cancers in an age-standardized population has steadily declined since the 1990s, the actual number of cancer diagnoses has increased by $73 \%$ since 1990 (1). This increase is a consequence of a growing population, the higher incidence of cancer in an aging population, and diagnosis of cancers through screening that would never have caused symptoms or death during a patient's expected lifetime. Cancer-related deaths in the United States have increased more modestly, by $29 \%$, from 469,000 in 1990 to 606,000 in 2020 (1). Thus, many more patients are living with a diagnosis of cancer. Against this backdrop, it is almost certain that cancer imaging will remain a major application of nuclear medicine in the coming years.

However, the way imaging studies are interpreted will likely change significantly. Currently, interpretation of oncologic imaging studies is almost exclusively a manual, subjective process: welltrained nuclear medicine physicians or radiologists scroll through endless stacks of images to identify and describe abnormalities. The rapid progress of machine learning-based approaches for image classification suggests that this process will soon be facilitated or maybe even taken over by computer systems (2). Nuclear oncologic imaging studies seem particularly well suited for AI-based interpretation because of the generally high contrast between tumor and normal tissue. This is already the case for tracers in clinical use, such as ${ }^{18} \mathrm{~F}$-FDG, somatostatin analogs, and prostate-specific membrane antigen (PSMA) ligands. Newer imaging agents for targets with more restricted expression in normal tissues, such as fibroblast activation protein (FAP) inhibitors, lend themselves even more to automatic image interpretation because of their yet higher tumorto-normal-tissue uptake ratios. 
Currently, oncologic imaging is heavily dominated by staging and restaging of patients before and after therapeutic interventions. Patients are imaged to determine whether there is metastatic cancer and whether the metastatic burden has decreased or increased in response to therapy. The success of theranostics has already changed this paradigm. Patients are no longer imaged solely to determine whether there is metastatic cancer but also to determine whether the cancer cells express a specific therapeutic target. For somatostatin receptor and PSMA expression, the consequence is targeted radionuclide therapy. However, in the future, the theranostic approach will probably be used in a broader sense and guide other therapies such as antibody-drug conjugates. Therefore, new tracers may be introduced primarily as companion diagnostics, and their application for staging and restaging will be a secondary application.

In oncologic research, studying the pharmacokinetics of therapeutic agents with nuclear imaging has been a goal for several decades. Major challenges have been the complexity of radiolabeling pharmaceuticals in due time for clinical use, as well as the limited sensitivity of PET scanners, which allowed imaging studies for only a few hours after drug administration. In recent years, there has been significant progress that may overcome both limitations. New totalbody systems show unprecedented sensitivity and increase the time window severalfold for pharmacologic studies (3). In parallel, antibodies have been incredibly successful for treatment of virtually all malignancies. In contrast to the labeling of small molecules, antibody labeling is a standardized process that can be applied irrespective of the antibody target. Thus, the combination of more sensitive PET systems with radiolabeled antibodies will provide many opportunities for oncologic research (4). Research with radiolabeled antibodies will likely identify new targets. Currently, the slow blood clearance and, hence, long circulation times of antibodies, necessitating the use of long-lived isotopes for labeling, leading to high radiation doses, remain limitations for antibody imaging in clinical routine. As long as these limitations are not overcome, imaging of such new targets with smaller molecules will remain more efficient. However, first steps, such as pre-targeting and novel antibody labeling methods, appear promising for reducing the radiation burden and for shortening the time between antibody injection and imaging (5).

Enormous investments have been made in the genomics, epigenetics, and proteomics ("omics") of the tumor tissue, as well as in analyses of circulating tumor cells and circulating tumor DNA. These technologies provide a wealth of information about cancer cells and are critical in selecting patients for specific therapies such as epithelial growth factor receptor and B-raf kinase inhibitors. Nevertheless, omics technologies lack spatial information, both on a microscopic level (e.g., intratumoral heterogeneity of cancer cells) and on a whole-patient level (e.g., location of metastases and intrapatient heterogeneity of metastatic lesions). Therefore, combining molecular imaging with omics technologies and analyses of circulating tumor cells seems to be a necessary step to advance our understanding of tumor biology and develop novel therapeutic strategies.

\section{MOLECULAR IMAGING IN ONCOLOGY}

\section{Carolyn J. Anderson, Michael M. Graham, and Jan Grimm}

Within the past few decades, oncologic imaging has been geared mostly toward targets on tumor cells. However, it has recently become quite clear that we were looking at only one part of the equation. We have left out of our sight a big part of what constitutes a tumor - the tumor microenvironment (TME) (Fig. 1). It comprises multiple different cell types, including immune, vascular, and stromal cells. Since these constitute a considerable proportion of a tumor, they are quite important and are a major factor in the overall prognosis, particularly in light of novel therapies such as checkpoint inhibitors and antivascular therapies. The future will therefore certainly spotlight this underexplored part of tumor biology, quite literally focusing on its components with radiolabeled tracers as well as with optical imaging. Using light, we can explore the microenvironment ranging from microscopic to macroscopic with multispectral imaging, allowing for multiplexed imaging of the various components of the TME (6). Although most optical imaging modalities, such as bioluminescence and microscopy, are confined mostly to the preclinical arena, a new modality has been developed that merges preclinical and clinical imaging in the form of optoacoustics, where pulsed light causes ultrasound signals from absorbers. Using intrinsic absorbers, we can explore the tumor microvasculature and its response to therapies or look at the distribution of fats and carbohydrates in tissues. Labeling of cells enables tracking of cellular therapies and the subsequent consequences on the tumor and its stromal components.

PET imaging of the ever-changing TME after immune modulation therapy has emerged as the next frontier in oncologic molecular imaging. The improved accuracy of predicting the outcomes of immunotherapy with ${ }^{89} \mathrm{Zr}$-labeled antibodies against programmed death ligand 1 and CD8-positive T cells for PET imaging demonstrates the promise of these strategies, which have been shown to be superior to biopsy and immunohistochemistry (7). The world of ${ }^{89} \mathrm{Zr}$-labeled antibodies for imaging immune cell markers is ever expanding and limited only by the targeting

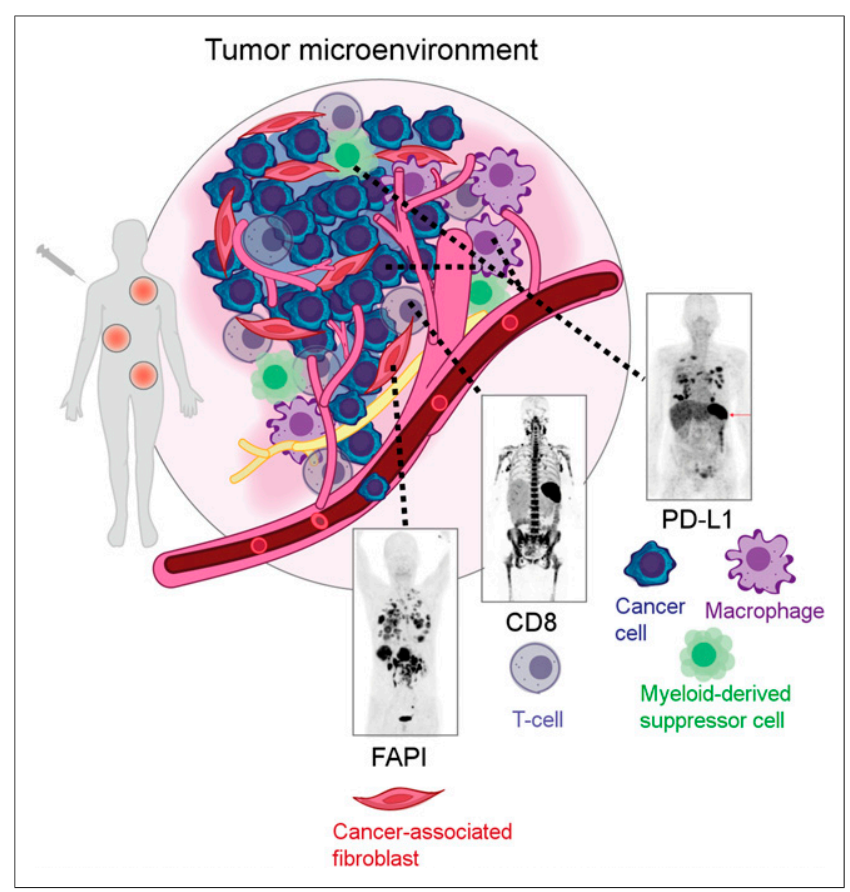

FIGURE 1. PET imaging of immune cell types in TME represents future direction of molecular imaging. Examples include imaging of cancer-associated fibroblasts (FAP inhibitor [FAPI]), CD8-positive T cells, and programmed death ligand 1 (PD-L1), which is found in antigen-presenting cells, including macrophages and myeloid-derived suppressor cells. (Art in Figure 1 courtesy of Lydia Perkins, PhD [Carnegie Mellon University, Pittsburgh, Pennsylvania.]) 
antigens and their expression levels. Preclinically, immuno-PET will continue to grow; however, translation will be limited to more widely applicable agents. Another attractive approach for imaging the TME will be focused on small-molecule or peptide imaging agents. These can be used on their own, such as FAP inhibitors to target the tumor stroma (8); can be combined with reporter genes transfected into cell-based therapies (e.g., PSMA-targeting CAR-T cells), which can attack both tumor cells in prostate cancer but also the PMSA-expressing tumor neovasculature; or can be incorporated into pretargeted approaches (e.g., bispecific antibodies or bioorthogonal click chemistry) (9).

Newly developed approaches, such as TME assessment with FAP inhibitor and immune modulation therapy assessment with in vivo T-cell labeling and with radiolabeled checkpoint inhibitors, will require Food and Drug Administration (FDA) approval, likely by a route different from the usual phase 1-2-3 scheme used for conventional drugs. The need for a new route arises because of the breadth of possible utility of these approaches: FAP inhibitor can detect a large variety of tumors (8), and immune checkpoint inhibitors are used to treat a wide variety of tumor histologies (10). The straightforward approach of performing clinical trials for a single tumor type with a specified therapy is inherently limiting, and to extend the label indications to other tumors will require multiple trials and years of effort. Thus, it is essential that the developers meet with the FDA to define an efficient and cost-effective way forward before starting trials. One approach might be to perform clinical trials that include - at the same time-TME imaging biomarkers commonly occurring in several types of tumors and therapies, with the hypothesis that if it works similarly with all, it should be widely applicable. At the same time that the discussion with the FDA is initiated, similar discussion with the Centers for Medicare and Medicaid Services should occur. It is essential that the trial design be acceptable to the Centers for Medicare and Medicaid Services as well as the FDA if new molecular imaging agents are to become widely distributed and clinically useful. The final step in developing molecular imaging agents is to ensure continued reimbursement. Currently, pass-through reimbursement stops after 2 years, when all the charges associated with a study are bundled. This method has usually resulted in reimbursement below cost. However, there are now ongoing legislative efforts to improve reimbursement, with a strong likelihood of success. This improvement should result in a more favorable environment for the development and use of new molecular imaging agents that will have a positive impact on human health in the future.

\section{THERANOSTICS}

\section{Lisa Bodei, Michael M. Graham, and Ken Herrmann}

With the introduction of PET, and later hybrid imaging including SPECT/CT, PET/CT, and PET/MRI, nuclear medicine instrumentation has progressed over the last 2 decades. The more recent clinical translation of theranostics beyond thyroid treatment has triggered a dramatic transformation of our field $(11,12)$. The identification of extra- and intracellular structures that can be targeted with radioactively labeled peptides, with small molecules, with antibodies, or with antibody fragments that can be exploited diagnostically and therapeutically will further expand theranostic applications.

Theranostics began with Saul Hertz in 1941 with the successful treatment of malignant and benign thyroid diseases with radioactive iodine, the first theranostic radiopharmaceutical (13). More recently, work in theranostics has accelerated, with somatostatin analogs labeled with various isotopes having been introduced diagnostically and therapeutically for the treatment and management of neuroendocrine tumors (14). Peptide receptor radionuclide therapy has become a major component in the management of unresectable or metastatic gastroenteropancreatic, bronchopulmonary, and other neuroendocrine tumors. Excellent safety profiles, disease control rates of $68 \%-94 \%$, quality-of-life improvements, and survival benefits have been reported (14). Importantly, ${ }^{177} \mathrm{Lu}$-DOTATATE was shown to be more effective than high-dose long-acting-release octreotide in midgut neuroendocrine tumors, which led to regulatory approval (15).

The success of peptide receptor radionuclide therapy provided the impetus to explore and commercially exploit the clinical value of PSMA-targeted theranostics. PSMA-directed PET imaging is now an important component of major clinical guidelines for detection of biochemical recurrence (16). A new-drug application for ${ }^{68} \mathrm{Ga}$-PSMA11 was recently submitted to the FDA, and approval is expected in the near future. Registration trials for ${ }^{18} \mathrm{~F}$-labeled PSMA ligands are ongoing (12). A prospective, randomized phase III study of ${ }^{177} \mathrm{Lu}$-labeled PSMA-617 has completed accrual, and results are expected soon. A significant improvement in progression-free survival would result in wide-ranging changes in the practice of nuclear medicine requiring adaptations of training curricula and creation of outpatient treatment centers.

At the same time, several other theranostic pairs are under investigation. One targets the chemokine receptor 4 and is currently undergoing clinical translation. Lead indications under investigation are hematologic malignancies such as multiple myeloma, leukemia, and central nervous system lymphoma. Phase I and II clinical trials are actively recruiting and enrolling patients $(11,12)$. Another attractive target is FAP, which is highly expressed in cancer-associated fibroblasts of the tumor stroma and in some cancer cells. FAPdirected theranostics will enable imaging of FAP expression with PET (Fig. 2) and targeting of the tumor stroma for treatment (8). When tumor cells, such as some soft-tissue sarcomas, also express FAP, a therapy with a FAP ligand alone may be effective. However, most cancer cells do not express FAP. Combination therapies that target the tumor stroma with FAP inhibitors and cancer cells with pharmacologic approaches may be required to achieve more profound therapeutic effects in most cancers (12).

Other potential theranostic targets include integrins (e.g., $\alpha_{\mathrm{v}} \beta_{6}$ integrin) that are overexpressed in a variety of poor-prognosis cancers (12) and melanocortin subtype 1, a highly expressed receptor in most melanomas (17). Both targets are being actively explored in ongoing early-phase clinical studies.

The next major advance in theranostics will be the introduction of $\alpha$-particle-emitting isotopes (18). Because of fundamental radiobiology differences, $\alpha$-particle radiation is considerably more effective than $\beta$. Recent preliminary reports from Germany on ${ }^{225}$ Ac-PSMA are encouraging (Fig. 3), and several groups are beginning human studies with other $\alpha$-emitting isotopes and other ligands (19).

As a prerequisite for future successful clinical translation, properly designed prospective, randomized trials need to be conducted and published in leading medical journals (9). In addition, radiation dosimetry and genomic assessment of radiosensitivity will guide precision theranostics to avoid both undertreatment and off-target toxicity. Although activity reductions may be required in some patients, much higher and potentially much more effective tumor doses may be achievable in many other patients. Phase 3 clinical trials exploiting 


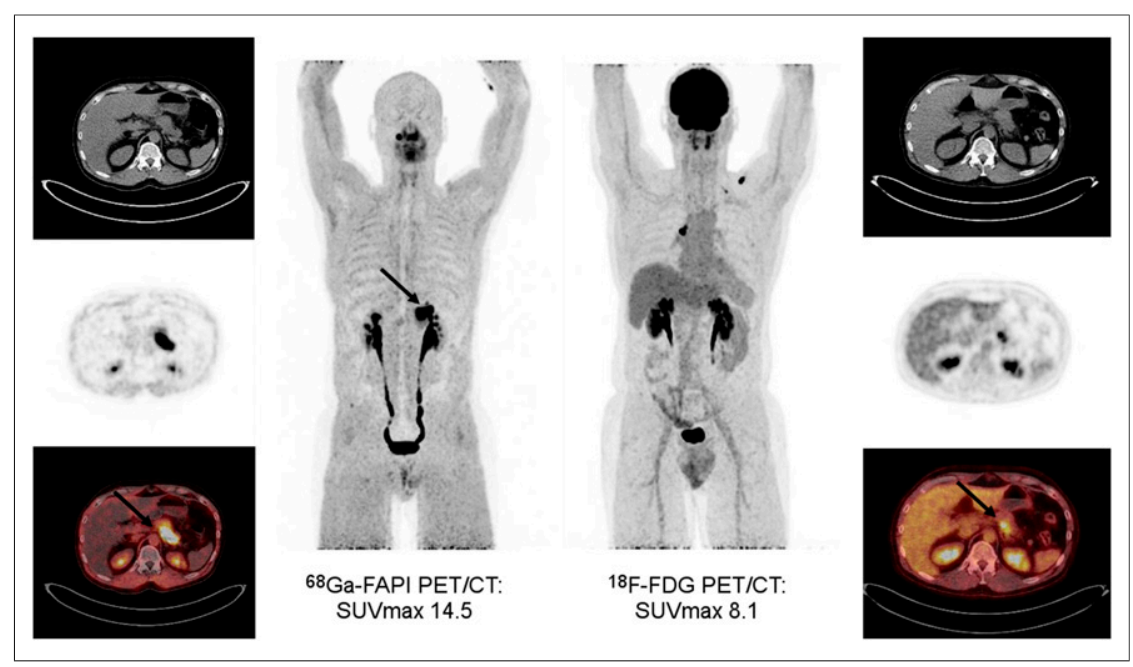

FIGURE 2. Patient with biopsy-proven pancreatic cancer (initial diagnosis made in May 2019) undergoing chemotherapy with gemcitabine/cisplatin (carbohydrate antigen 19-9 level $>4,000$ $\mathrm{U} / \mathrm{mL}$ ). Corresponding ${ }^{68} \mathrm{Ga}-\mathrm{FAP}$ inhibitor (FAPI) and ${ }^{18} \mathrm{~F}-\mathrm{FDG}$ PET/CT images are displayed (axial CT [top], PET [middle], and PET/CT [bottom], as well as whole-body PET/CT [center]). Intense focal FAP inhibitor uptake but only moderate ${ }^{18} \mathrm{~F}$-FDG uptake is seen in pancreatic tail (arrows).

target visualization with PET and individualized therapies will help to realize the full potential of clinical theranostics.

\section{NEUROLOGY}

\section{Henryk Barthel}

In the field of molecular brain imaging, it is expected that several current research applications will transform into routine clinical tools in the coming years. This transformation refers to both radiotracer developments and technical advancements. In addition, new and promising research approaches will emerge.

As an example for a successful transfer of research from bench to bedside, there is reason to believe that in the future we will have brain PET tracers to visualize most, if not all, neurodegenerative disorders. This development started with the approval of the known $\beta$-amyloid PET tracers some years ago and continued with the first approved tau PET tracer (20). It will hopefully also include PET tracers of $\alpha$-synuclein, TDP43, ubiquitin, and other protein aggregates, as they represent histopathologic hallmarks of different dementia and movement disorders. As such, it will be possible to shift the time point of accurate diagnosis of these disorders from post mortem to ante mortem, that is, to a time point at which the diagnosis can (provided that corresponding treatment approaches are available) impact the patient outcome.

This shift may even be applicable to the prodromal or presymptomatic disease stages: PET imaging will be used as standard clinical care to elucidate underlying pathology with high sensitivity and many years before the first cognitive or motor symptoms occur. This early imaging biomarker approach will likely be used in subjects at risk (e.g., in those with rapid-eye-movement sleep disorder in cases of Parkinson disease, or in those with subjective cognitive impairment in cases of Alzheimer disease $[\mathrm{AD}]$ ) or even as a follow-up to regular blood screening at certain age levels. Doing so will open - for the first time - a window of opportunity for preventive treatments in these devastating disorders.

It is anticipated that the current definitions and diagnostic approaches to neurodegenerative disorders will be subjected to scrutiny, with major expansions in the clinical use of molecular brain imaging. This paradigm shift is mainly motivated by the fact that for all of these disorders and the way they are currently diagnosed it was so far not possible to provide disease-modifying treatments. With $\mathrm{AD}$ as a primer, it was recently proposed to replace the current definition of the disease as a syndromal construct by defining it on biologic grounds, that is, by carrying out diagnostic tests for amyloid, tau, and neurodegeneration (the so-called ATN concept) (21). As soon as targeted therapeutic agents are successful, this paradigm shift-which puts PET imaging and other molecular diagnostics into the driver seat - can be expected to be applied to other neurodegenerative diseases (Fig. 4).

In line with this predicted paradigm shift on how to diagnose neurodegenerative diseases, there is growing evidence that PET imaging will gain a larger role in the context of treatment stratification and therapy monitoring. For antiamyloid drugs in $\mathrm{AD}$ as the most advanced disease-modifying therapeutic approach in neurodegeneration, there is optimism that the human monoclonal antibody aducanumab will be approved for clinical use (22). Because such drugs will be quite expensive and might have relevant side effects, experts predict that a positive amyloid PET scan proving the presence of the actual drug target will be required to justify drug administration. Such an application is reasonable, on the basis of the experience obtained in drug-testing trials on 


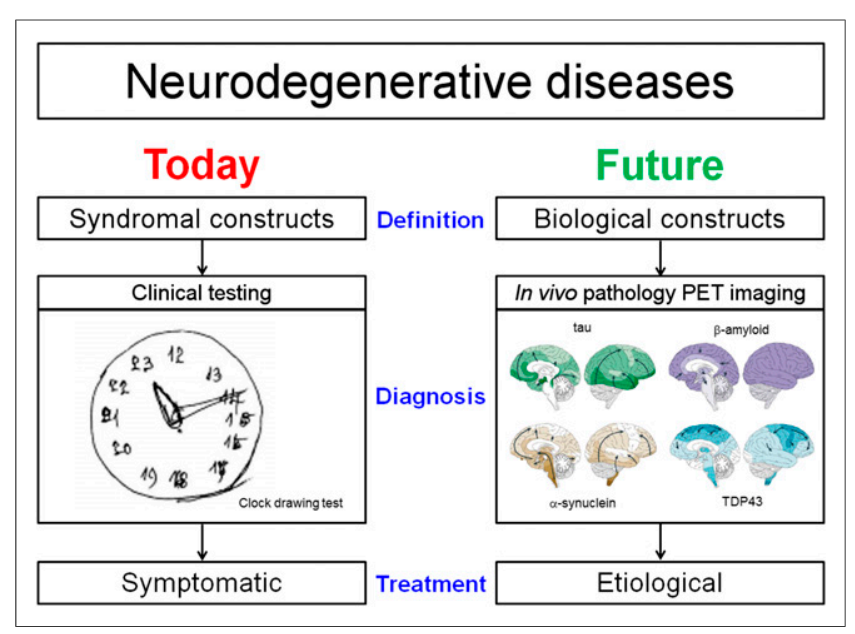

FIGURE 4. Schematic presentation of expected paradigm shift in how neurodegenerative diseases are defined, diagnosed, and treated, together with future role of PET imaging in this context. TDP43 = TAR DNA-binding protein 43. (Illustrations of in vivo pathology PET imaging reprinted from Brettschneider et al. (50) with permission by Springer-Nature.)

patients with a clinical diagnosis of probable AD. Despite standardized assessment by expert clinicians, baseline amyloid PET findings were negative in about $20 \%$ of these patients (23). The percentage of incorrect diagnoses will increase as the treatment paradigm moves into earlier disease: only $50 \%$ of subjects with mild cognitive impairment are amyloid-positive. Further, as - with increasing patient agethe probability of multiple underlying neuropathologic conditions increases, multitracer PET might well become the tool of choice for planning individualized treatments and monitoring therapy. In this way, we can think for the first time about tailored, individually targeted interventions for patients with neurodegenerative diseases.

The coming years also expect to see more research on other exciting targets for molecular brain imaging. With that expectation, we hope for answers on questions such as whether synaptic density PET imaging will find a clinical application, whether myelin PET imaging will fulfill the expectation of revolutionizing the diagnosis of multiple sclerosis, whether we will see a clinical application for PET imaging in psychiatric conditions, and, if the answer is yes, whether that application will be neuroinflammation imaging.

Finally, with regard to technical advancements, we will certainly see more research in the years to come addressing the important question of the clinical value (despite the proven value in research) of hybrid PET/MRI and AI in molecular brain imaging. For hybrid PET/MRI, many experts predict that $\mathrm{AD}$ will be the first clinical application once disease-modifying drugs are available. For AI, there is hope to, for instance, better differentiate disease subtypes, with implications for improved differential diagnosis and management decisions.

Taken together, the future is bright for molecular brain imaging, both in research and in clinical practice. Also, as is of special relevance to many brain disorders-and in view of the increased economic pressure to justify the clinical use of new diagnostic approaches-we should not forget the intrinsic value of a reliable diagnosis to our patients and their caregivers. In the face of the continued improvement of competing modalities, the challenge is to ensure that nuclear medicine continues to provide high-quality regional quantitative brain state information not otherwise obtainable to guide patient treatment.

\section{CARDIOLOGY}

\section{Frank Bengel, Marcelo DiCarli, Heinrich Schelbert, and H. William Strauss}

Cardiovascular applications of nuclear medicine have focused on assessments of myocardial perfusion, function, and viability in ischemic heart disease, to guide revascularization interventions. Despite steady progress in imaging technology, true molecularly targeted imaging has not yet contributed to clinical cardiovascular medicine in a manner similar to oncology or neurology. This difference is best explained by the fact that cardiology has so far been dominated by mechanical interventional therapies and broadly applicable, generalizable drug therapies, in which targeted molecular imaging-which works best in combination with targeted molecular therapies-has not necessarily been needed.

The future of cardiovascular nuclear medicine, however, will be driven by molecular imaging. This will be tightly linked with the future development of cardiovascular therapy, in which molecularly targeted strategies will play an increasing role (24). The recent success of radionuclide imaging for the detection of cardiac amyloidosis is a prime example that molecular imaging will achieve clinical relevance when targeted therapies define a specific need (25). For this rare but frequently fatal disease, various highly specific but also costly targeted drugs have recently emerged and require precise identification of the most suitable candidates. Similarly, other specific therapies_-including RNA products; targeted peptides; or small molecules, nanoparticles, or even cell products-are under development for various pathologies of the myocardium, vessel wall, conduction system, or valves. Those will ultimately require guidance by tissue-specific biomarkers. In the field of cardiac repair, for example, modulation of the immune system and of tissue fibrosis is actively pursued. Here, targeted imaging will serve as the key biomarker for identification of the most suitable patients and for monitoring of treatment success. Ultimately, even the theranostic principle, by which the target of a molecular intervention is directly visualized by molecular imaging, will become a reality in cardiovascular care. Today, chemokine receptors such as CXC-motif chemokine receptor 4 (CXCR4) or CC-motif chemokine receptor 2 (CCR2), and profibrotic factors such as FAP are readily visualized by radionuclide imaging. Notably, they are also targets for cardiovascular tissue repair in experimental conditions. Tomorrow, these advances in imaging and therapy will be merged for image-guided precision care in well-selected clinical scenarios, and they will serve as a role model for the implementation of further, similar strategies throughout clinical cardiology.

Increasingly, radiopharmaceuticals that were originally designed for other applications turn out to be of value for cardiovascular imaging. Concurrent examples include the use of bone-seeking agents or $\beta$-amyloid-targeted agents for cardiac amyloidosis detection, the use of sodium fluoride for imaging of atherosclerosis and valvular calcification, or the use of several tumor-binding agents such as DOTATATE or methionine for imaging inflammation in the vessel wall and myocardium. On the one hand, these examples show that between different areas of nuclear medicine there is crossfertilization, which will be increasingly recognized and exploited. On the other hand, these and other examples emphasize the notion that molecular pathways of disease are frequently not restricted to a single organ or lesion. Similarities between organs, their interaction, or their heterogeneity in various tissues, along with their identification using imaging biomarkers, will be increasingly relevant in the era of precision medicine. 
Finally, cardiovascular medicine will be increasingly shaped by systems-based, holistic approaches that recognize that the heart is at the center of the circulatory system but also is affected by various other systems, such as the neurohumoral or immune system. Thereby, cardiovascular conditions are tightly linked with disease of other organs and tissues, and any systemic, targeted intervention should consider the entirety of effects on the target region and other tissues. Because radionuclide-based molecular imaging is a whole-body technique, it is uniquely suited to uncover such systemic networks. The inflammatory link between heart, hematopoietic system, vessel wall, and other organs such as brain and kidneys is one timely example (26). Likewise, the recent emphasis on imaging the effects of tumors and tumor therapy on the cardiovascular system (cardiooncology) is another example (27).

The future of cardiovascular nuclear medicine will therefore be shaped by novel, targeted imaging techniques that will be designed to guide specific molecular interventions, based not only on the individual biology of the cardiovascular target tissue but also on the concomitant state of networking remote organs and tissues. This guidance will lead to tailored precision therapy that respects the entire body as a networking system, leading to optimal cardiovascular outcome paralleled by minimal harm and maximal benefit for other organs.

\section{RADIOPHARMACEUTICAL CHEMISTRY}

\section{Carolyn J. Anderson and Jason S. Lewis}

Radiopharmaceutical chemistry has evolved dramatically into a multidisciplinary field in which radiochemists work with biologists, physicists, mathematicians, engineers, and, of course, clinicians from many disciplines.

Although fundamental knowledge about radiochemistry has burgeoned (28), the translation of basic radiochemistry discoveries into clinical advancements can still be slow and inefficient. Radiochemists are currently pursuing new, quicker, and more practical ways to produce radiolabeled ligands for the next generation of molecular targets. The development of new radiotracers for PET imaging (in oncology, cardiology, neurology, infection, inflammation, and others) and theranostics will require close collaboration with physicians to identify and prioritize clinical needs and unanswered questions and with biologists from all disease fields to best choose molecular targets for radiopharmaceutical development.

In the past few decades we have seen a resurgence of new methods for the labeling of molecules with ${ }^{11} \mathrm{C}$ and ${ }^{18} \mathrm{~F}$; the application of radiometals - most notably ${ }^{64} \mathrm{Cu}$ and ${ }^{89} \mathrm{Zr}$ - has perhaps seen the most growth in terms of basic, translational, and clinical studies. In the next decade, we must continue to develop new chemical reactions and pathways that overcome synthetic constraints related to working with short-lived radioisotopes at high specific activity. We need to develop versatile, reproducible, and translatable radiotracer chemistry methods for routine nuclear medicine applications. This development requires the continued creation and improvement of modular automation technology for good-manufacturing-practice radiopharmaceutical synthesis that is adaptable to a variety of reaction scenarios. One of the major impediments in the pathway to translation has been the general lack of "kit"-like formulations, which can be used with a variety of chelators and metals and thereby aid in the production of radiolabeled antibodies or peptides under good-manufacturing-practice-like conditions. Notwithstanding significant strides already being made in the ${ }^{68} \mathrm{Ga}$ arena, this should be an area of focus in the coming years.
Future developments will perhaps be centered on the use of $\alpha$-emitters, given the exciting clinical results with ${ }^{225}$ Ac-PSMA (19). Collaboration with radiobiologists will be required to understand the biologic behavior of $\alpha$-emitting radionuclide daughters and to leverage them for therapy or for mitigation of toxicity. In the context of metal- and halogen-based $\alpha$-emitters, novel conjugation methods and chelators that are universal for imaging-therapy radionuclides - the theranostic nuclide pairing paradigm — will likely become an exciting area for future development.

The radiochemistry field will also expand if parallel advances are made in the identification of new imageable targets: recent work has shown that even antigen targets of low abundance can be imaged with the correct platform (29). In some ways, this development goes against longstanding doctrine; many targets that were previously assumed to be inappropriate could be reexamined, particularly with the recent advances in highly sensitive whole-body PET scanners (3).

In the oncology setting, radiopharmaceuticals have been shown to effectively demonstrate target engagement, such as in the use of ${ }^{18}$ F-fluoroestradiol in the context of a novel estrogen receptortargeted therapeutic (30). A well-designed tracer could also be used to noninvasively monitor the effectiveness of therapeutic drugs downstream of the drug target. One such example is the use of human epidermal growth factor receptor 2 imaging for agents that target tyrosine kinase and, downstream, inhibit human epidermal growth factor receptor 2 (31). The clinical applications of such radiotracers have significant implications for use in phase I and II clinical trials and for streamlining the approval process for novel therapeutics and radiotherapeutics.

These developments depend on the availability of radionuclides. Radiochemists also discover novel approaches to produce radionuclides for imaging and therapy; it is therefore imperative that federal agencies and commercial entities continue to support new production and isolation routes for isotopes. The ${ }^{99 \mathrm{~m}} \mathrm{Tc}$ shortages underscore the necessity to support radiochemists in their pursuit of simpler and less expensive routes to radioisotope production and isolation in order to increase availability, reduce costs, and ensure the reliability of the supply. Achieving this goal would, in turn, allow for increased numbers of clinical trials and more agents receiving FDA approval.

Finally, the future of radiopharmaceutical chemistry depends on training the next generation of scientists who must speak multiple scientific languages, with chemistry and radiochemistry being a primary focus. There is also a critical need for well-trained translational radiochemists who can bridge the divide between basic research enterprises and clinical investigation. The shortage in radiochemistry expertise is a critical issue and, if not solved, will fundamentally limit advances not just in radiochemistry but nuclear medicine as a whole. Addressing this shortage also requires support for the development of expert radiopharmacists who are familiar with clinical research and the regulatory pathways for investigational new drug application and approval. It is clear that we need innovative educational programs with a specialized training curriculum derived from collaboration across multiple disciplines if this field is to continue to confidently advance into the future.

\section{INSTRUMENTATION AND DATA ANALYSIS}

David A. Mankoff, Ramsey D. Badawi, and Todd E. Peterson

The first 60 years of The Journal of Nuclear Medicine have witnessed extraordinary developments in nuclear medicine instrumentation and 
data analysis that have helped drive the field's advancing science and clinical impact. Nuclear imaging detectors have evolved from single-channel probes scanned in a rectilinear fashion to sophisticated position-sensitive detectors with high resolution in energy, space, and time. Imaging systems have evolved from purely planar cameras to tomographs capable of generating high-quality 3- and 4-dimensional data. PET and SPECT devices were combined with $\mathrm{CT}$ and later MRI to provide precisely aligned anatomic information to complement the functional and molecular information provided by nuclear imaging. These improvements in instrumentation were accompanied by parallel advances in mathematics and computing for PET and SPECT image reconstruction and analysis. The development of iterative image reconstruction methods based on statistical and physical models, now the standard for both PET and SPECT, ushered in a new era of image quality. In addition to generating images, our ability to measure regional tracer concentration improved notably, first for PET and more recently for SPECT, along with advanced image analysis methods including pharmacokinetic models and novel data analysis methods that can quantify complex molecular processes such as enzymatic activity and receptor binding.

What instrumentation and data analysis advances will the next 60 years of The Journal of Nuclear Medicine bring us? The following accomplishments, highlighted in the Journal over the past several years, provide an exciting indication of what is to come:

Detectors. Advances in material science and electronics have led to faster, more compact, and more accurate detectors (32). Fast scintillators such as lutetium-yttrium oxyorthosilicate have enabled timing resolution that supports time-of-flight annihilation photon detection PET, now the clinical PET standard that yields improved image quality and quantitative accuracy. The development of highly compact solid-state devices to convert scintillation photons to electrical signals-often called silicon photomultipliers-has led to improvements in detector compactness, accuracy, and timing. These and similar devices have also enabled imaging in high magnetic fields in PET/MRI, not possible with standard photomultipliers. Solid-state devices also enable direct detection of high-energy photons, such as in the cadmium zinc telluride detectors used for SPECT. Overall, these advances have heralded the emergence of digital detectors as the standard for nuclear imaging devices. We are likely to see further advances in performance-particularly in timing resolution-and reductions in cost in the future, paving the way for new applications and broader implementation.

Devices. Advances in detectors, processing electronics, and reconstruction algorithms have supported the ability to build totalbody PET devices with a long axial field of view (33). The fact that these devices can simultaneously detect nuclear emissions throughout the body leads to extraordinary photon sensitivity, facilitating imaging with very low levels of radioactivity or ultrashort imaging times. Total-body PET offers an unprecedented ability to simultaneously measure whole-body tracer uptake and kinetics and, thus, to measure integrated molecular biology - that is, systems biology-that is applicable to diagnosis, pharmacology, and biologic discovery. Early results of such imaging at 2 U.S. centers - the University of California Davis and the University of Pennsylvania-provide examples of these new devices' capabilities, which include whole-body dynamic imaging and imaging performed at more than 10 isotope half-lives after injection. Bringing devices with a longer axial field of view to research and the clinic will be an important component of future work.
Image Generation and Analysis. As devices increase their ability to acquire data, there will be an increasing need for fast, efficient reconstruction algorithms that can leverage advancing computing methods, including cluster and cloud computing. Advances in data science, including machine learning and AI, may provide avenues for increased efficiency and accuracy in image generation. Similarly, the integration of biokinetic tracer models and patient motion models into the physical models used for image reconstruction may add to the efficiency and accuracy of image reconstruction algorithms. Improvements in imaging devices and image generation algorithms have yielded imaging data of higher quality and quantity, creating a rich environment for harvesting biologic and clinical insights to generate molecular imaging biomarkers (34). Novel approaches for assessing patterns of static uptake on 3-dimensional images and improved 4-dimensional kinetic analysis-including emerging radiomics- and AIbased methods-provide a wealth of methodologies by which to harvest the unique information on in vivo biology that can be derived from molecular imaging. A closely related and complementary partner to image analysis is accurate radiopharmaceutical dosimetry for theranostics (35).

Standards for Quantitative Imaging. The inherent ability of nuclear imaging to measure regional radiopharmaceutical concentration creates the opportunity for quantitative image biomarkers for diagnosis and treatment guidance. However, variability in approach has limited the application of quantitative molecular biomarkers to clinical practice. Setting standards for quantitative molecular imaging has been the goal of published criteria such as PERCIST for ${ }^{18}$ F-FDG PET and cancer (36) and of organizations focused on quantitative imaging in the United States and Europe. Furtherance of these efforts will be important to leverage the full power of quantitative molecular imaging in clinical practice.

Instrumentation for Nonnuclear Molecular Imaging. As clinical molecular imaging expands beyond nuclear methods, advances in nonnuclear instrumentation and image generation methods will play an important role in translation of these methods from preclinical studies to the bedside. These advances includes those in optical tomography, optoacoustic imaging (37), ultrasound imaging of targeted contrast media, and MR spectroscopy, including hyperpolarized MRI agents. Advances in targeted radiographic contrast media might also bring molecular imaging capabilities to $\mathrm{CT}$, with the aid of advanced instrumentation methods such as photon-counting CT. These advances will support the translation of multimodality approaches needed to measure complex biologic processes such as metabolism and immunology.

These are but a few of the areas of research in instrumentation and data analysis that are likely to be important in the next 60 years of The Journal of Nuclear Medicine to help shape the evolving and increasing role of molecular imaging in biologic research and precision medicine.

\section{Al}

\section{Irène Buvat}

$\mathrm{AI}$ is currently reshaping many research and development fields, and nuclear medicine and molecular imaging are no exception. The promise of AI and neural network-based technologies in the nuclear medicine arena for image processing and pattern recognition was recognized in the early 1990s $(38,39)$, supported by proof-of-concept papers in the cardiac and brain imaging domains $(40,41)$. Yet, it took 25 years for this promise to mature and start 
becoming a clinical reality-not until the tremendous progress that was made in AI techniques, with the first AI-based products now being offered as part of the vendors' equipment and software with 3 flavors: automation of some image acquisition settings (e.g., patient positioning and scanning time), production of highquality quantitative images (e.g., using AI-based scatter, attenuation and motion corrections, image reconstruction, or denoising), and image analysis and interpretation. Upstream, AI will certainly improve the efficiency of the tracer development process, although first results in that area are yet to come.

Automation of the settings associated with the acquisition process is already transparent to the users, who may not even be aware that this automation relies on AI. Improvements will continue in that direction to ensure optimized settings for each patient and faster workflow.

AI-driven corrections, image reconstruction, and denoising will produce enhanced images and will soon be provided by vendors as components of the scanners. The black-box problem associated with many AI algorithms will not be an issue in that context, provided proper quality control procedures are used for scanner acceptance testing. Although the underlying AI algorithms will remain opaque for most nuclear medicine physician and physicist consumers, they will significantly change the practice by enabling shorter acquisition durations (or lower injected doses) and finer spatial and timing resolutions, hence producing sharp molecular images that will further broaden the fields of application. Insights about physiologic and pathologic mechanisms that we start gaining through the use of total-body PET scanners might actually be used to advantage when AI-based reconstruction algorithms are being trained. These algorithms would thus be able to produce total-body PET-like images from scanners that are modern but do not have an ultralong axial field of view. In that respect, AI might be a key component for leveraging technology investments (the same would apply to ultrafast PET detectors) to maximize their reach in nuclear medicine clinical practice. If so, what has always been considered a limitation of PET and SPECT imaging, namely modest spatial and temporal resolution, would be overcome and broadly available scanners will soon offer an unparalleled combination of high sensitivity and high spatial and temporal resolution.

AI is also changing the way nuclear medicine images are read and interpreted. Tedious and time-consuming tasks such as delineation of structures of interest will soon be fully automated thanks to AI algorithms trained on thousands of cases. Images will be displayed with all suggestive regions preidentified, thus considerably accelerating their visual analysis by nuclear medicine physicians and radiologists. Quantitative features, which nuclear medicine is good at providing, will be extracted and reported without the need to set landmarks or draw regions of interest. All these advances are within reach and, after clearance by authorized bodies, will be clinically available with great benefits: time savings, increased reproducibility, and quantitative reporting. More challenging but also within view is that, beyond picking up abnormalities, AI will detect disease and make predictions and classifications based on molecular imaging features with well-controlled and small error rates. Although encouraging results are being reported, some crucial elements are still needed before these AI-based prediction and classification models can gain wide acceptance and become routine tools assisting physicians in a broad range of applications. The first is that their robustness and clinical or economic value for patient management in multicenter settings need to be demonstrated-a goal that will be achieved within a few years for specific applications. The second is the need to exploit more fully the ability of the AI-driven models to integrate much more than the image content and basic clinical data, namely a wide variety of semantic and quantitative content of biologic, omics, histologic, comorbidity, or comedication data stored in patient records. Achieving this goal will require joint effort by different communities, specific data workflow management, and interoperability between information systems, along with the accompanying complex legal, technical, and scientific challenges. Yet, this data integration process, supported by AI, might be a game changer within the next 10 years. Indeed, AI is absolutely needed to assist human intelligence in this data integration process. For a given patient, we are currently collecting much more information than even several experts from different specialties together can comprehensively analyze in a reasonable time, accounting for not only all information brought by each data type but also previous knowledge learned through experience with many patients seen over the years. Automated mining of such complex data is unquestionably required to help us distinguish associations and draw connections not only between data collected from a single patient but also between data observed in many. Only AI algorithms can handle so many complex data of so many different kinds and highlight patterns that we can then turn into biologic hypotheses. AI is our best ally to work synergistically with complementary specialties and further understand the complexity of pathologic processes through the prism of exquisite molecular imaging augmented by other data types. Let us welcome AI in our field and make it grow further, not only by producing clearer images of increasingly refined molecular targets and facilitating their interpretation but also by enabling new molecular discoveries that will impact both basic science and patient care.

\section{CONCLUSION}

During the last 60 years, nuclear medicine has undergone many changes. Diagnostic tests that were once considered to be at the very heart of the discipline, such as colloid scans for detection of liver metastases, have almost entirely been replaced by other imaging studies. At the same time, new imaging technologies, such as PET, that were considered pure research tools have become routine clinical tests. Technologic innovations have occurred rapidly and often were unpredicted. At the beginning of the 2000s, many experts questioned, for good reasons, the clinical need for PET/CT systems (42). Conversely, SPECT/PET systems were considered to be a promising approach to make PET imaging more broadly available clinically (43). Nevertheless, standalone PET systems and combined SPECT/PET systems were no longer commercially available by the 2010s.

Similar observations can be made for nuclear imaging agents. Imaging of prostate cancer with radiolabeled PSMA inhibitors was believed not to be feasible, until the opposite was proven recently (44). Good arguments were also made that the development of new imaging agents is generally too expensive to be economically viable (45). Therefore, the whole field of radiochemistry and radiopharmacy seemed doomed, and the expectation was that for years to come, there would be no clinical PET imaging agent other than ${ }^{18} \mathrm{~F}-\mathrm{FDG}$. The FDA approvals of ${ }^{11} \mathrm{C}$ choline, somatostatin receptor, amyloid, and tau ligands, as well as the several ongoing registration trials for PSMA ligands, have proven these well-founded concerns wrong. 
However, the changes that nuclear medicine underwent go even deeper than the evolutions or revolutions of imaging equipment and radiopharmaceuticals. Nuclear medicine is commonly defined as the use of radioisotopes for diagnosing and treating human diseases. Yet, the relative importance of the diagnostic and therapeutic applications of nuclear medicine repeatedly changed. Nuclear medicine started as a part of internal medicine and was clinically applied mainly for therapy of thyroid disorders and for studies of the pathophysiology of various diseases $(46,47)$. During the following years, expansion of the therapeutic applications of nuclear medicine beyond thyroid disorders proved challenging. At the same time, however, the quality and spatial resolution of nuclear images improved, and consequently, the field moved closer to radiology. The introduction of PET/CT drastically accelerated this development. At the beginning of the 2010s, clinical nuclear medicine appeared so similar to radiology that the American Board of Nuclear Medicine proposed abandoning nuclear medicine as an independent medical specialty (48). The efficient reading of ${ }^{18} \mathrm{~F}-\mathrm{FDG}$ PET/CT scans was felt by many to be the only economically viable task for nuclear medicine physicians. Under this assumption, it seemed obvious that nuclear medicine should become a subspecialty of radiology. Only after intensive discussions with its stakeholders (49) did the American Board of Nuclear Medicine withdraw this proposal.

Recent developments indicate that this decision was the right one. Several new imaging agents have been FDA-approved in the interim, and it appears unlikely that nuclear medicine will be reduced to ${ }^{18} \mathrm{~F}$ FDG PET/CT reading. Even more important are the FDA approval of the theranostic pair ${ }^{68} \mathrm{Ga}$-DOTATATE and ${ }^{177} \mathrm{Lu}$-DOTATATE for imaging and therapy of neuroendocrine tumors, as well as the highly promising results for imaging and treatment of prostate cancer with radiolabeled PSMA ligands. Thus, it is highly likely that therapeutic applications of radioisotopes will play a key role in clinical nuclear medicine in the coming years. In other words, the field will move from nuclear radiology back to nuclear medicine.

Yet, as pointed out by several of the examples above, the future of nuclear medicine has been notoriously difficult to predict. Thus, we would not be surprised if many or all of our predictions turn out to be incorrect. In fact, we almost hope that our predictions are wrong. The development of nuclear medicine has been unpredictable because the field has been so innovative and able to quickly adapt to new scientific developments and clinical needs. If this innovation continues, there is no question that the future of nuclear medicine is bright.

\section{DISCLOSURE}

No potential conflict of interest relevant to this article was reported.

\section{ACKNOWLEDGMENTS}

Carolyn Anderson, Michael Graham, and Jan Grimm thank Lydia Perkins for the graphics in Figure 1. Henryk Barthel thanks John Seibyl and Victor Villemagne for fruitful discussions.

\section{REFERENCES}

1. Siegel RL, Miller KD, Jemal A. Cancer statistics, 2020. CA Cancer J Clin. 2020;70:7-30

2. Ardila D, Kiraly AP, Bharadwaj S, et al. End-to-end lung cancer screening with three-dimensional deep learning on low-dose chest computed tomography. Nat Med. 2019;25:954-961.

3. Badawi RD, Shi $\mathrm{H}$, Hu P, et al. First human imaging studies with the EXPLORER total-body PET scanner. J Nucl Med. 2019;60:299-303.
4. Lohrmann C, O'Reilly EM, O'Donoghue JA, et al. Retooling a blood-based biomarker: phase I assessment of the high-affinity CA19-9 antibody HuMab-5B1 for immuno-PET imaging of pancreatic cancer. Clin Cancer Res. 2019;25:7014 7023.

5. Houghton JL, Zeglis BM, Abdel-Atti D, Sawada R, Scholz WW, Lewis JS. Pretargeted immuno-PET of pancreatic cancer: overcoming circulating antigen and internalized antibody to reduce radiation doses. J Nucl Med. 2016;57:453459

6. Omar M, Aguirre J, Ntziachristos V. Optoacoustic mesoscopy for biomedicine. Nat Biomed Eng. 2019;3:354-370.

7. van de Donk PP, Kist de Ruijter L, Lub-de Hooge MN, et al. Molecular imaging biomarkers for immune checkpoint inhibitor therapy. Theranostics. 2020;10:17081718 .

8. Kratochwil C, Flechsig P, Lindner T, et al. ${ }^{68} \mathrm{Ga}$-FAPI PET/CT: tracer uptake in 28 different kinds of cancer. J Nucl Med. 2019;60:801-805.

9. Martinez O, Sosabowski J, Maher J, Papa S. New developments in imaging cellbased therapy. J Nucl Med. 2019;60:730-735.

10. Esfahani K, Roudaia L, Buhlaiga N, Del Rincon SV, Papneja N, Miller WH Jr. A review of cancer immunotherapy: from the past, to the present, to the future. Curr Oncol. 2020;27(suppl 2):S87-S97.

11. Langbein T, Weber WA, Eiber M. Future of theranostics: an outlook on precision oncology in nuclear medicine. J Nucl Med. 2019;60(suppl 2):13S-19S.

12. Herrmann K, Schwaiger M, Lewis JS, et al. Radiotheranostics: a roadmap for future development. Lancet Oncol. 2020;21:e146-e156.

13. Hertz B. A tribute to Dr. Saul Hertz. World J Nucl Med. 2019;18:8-12.

14. Bodei L, Kwekkeboom DJ, Kidd M, Modlin IM, Krenning EP. Radiolabeled somatostatin analogue therapy of gastroenteropancreatic cancer. Semin Nucl Med. 2016;46:225-238.

15. Strosberg J, El-Haddad G, Wolin E, et al. Phase 3 Trial of ${ }^{177} \mathrm{Lu}$-dotatate for midgut neuroendocrine tumors. N Engl J Med. 2017;376:125-135.

16. Cornford P, Bellmunt J, Bolla M, et al. EAU-ESTRO-SIOG guidelines on prostate cancer. Part II: treatment of relapsing, metastatic, and castration-resistant prostate cancer. Eur Urol. 2017;71:630-642.

17. Li M, Zhang X, Quinn TP, et al. Automated cassette-based production of high specific activity $\left[{ }^{203 / 212} \mathrm{~Pb}\right]$ peptide-based theranostic radiopharmaceuticals for image-guided radionuclide therapy for cancer. Appl Radiat Isot. 2017;127:5260 .

18. Seoane DC, De Saint-Hubert M, Crabbe M, Struelens L, Koole M. Targeted alpha therapy: a critical review of translational dosimetry research with emphasis on actinium-225. Q J Nucl Med Mol Imaging. 2020;64:265-277.

19. Kratochwil C, Bruchertseifer F, Giesel FL, et al. ${ }^{225}$ Ac-PSMA-617 for PSMAtargeted alpha-radiation therapy of metastatic castration-resistant prostate cancer. J Nucl Med. 2016;57:1941-1944.

20. FDA approves Tauvid as first tau protein tracer tool for Alzheimer's diagnosis. Alzheimer's News Today website. https://alzheimersnewstoday.com/2020/06/01/ fda-approves-tauvid-first-tau-protein-tracer-agent-alzheimers-diagnosis. Published June 1, 2020. Accessed August 4, 2020.

21. Jack CR, Bennett D, Blennow K, et al. NIA-AA research framework: toward a biological definition of Alzheimer's disease. Alzheimers Dement. 2018;14:535562.

22. FDA filing of Alzheimer's therapy aducanumab delayed to late 2020, Biogen says. Alzheimer's News Today website. https://alzheimersnewstoday.com/2020/ 04/27/fda-filing-of-alzheimers-therapy-aducanumb-delayed-to-late-2020. Published April 27, 2020. Accessed August 4, 2020.

23. Barthel H, Seibyl J, Sabri O. The role of positron emission tomography imaging in understanding Alzheimer's disease. Expert Rev Neurother. 2015;15:395-406.

24. Werner RA, Thackeray JT, Diekmann J, Weiberg D, Bauersachs J, Bengel FM. The changing face of nuclear cardiology: guiding cardiovascular care toward molecular medicine. J Nucl Med. 2020;61:951-961.

25. Masri A, Bukhari S, Eisele YS, Soman P. Molecular imaging of cardiac amyloidosis. J Nucl Med. 2020;61:965-970.

26. Thackeray JT. Imaging the molecular footprints of the heart-brain axis in cardiovascular disease. J Nucl Med. 2019;60:728-772.

27. Dreyfuss AD, Bravo P, Koumenis C, Ky B. Precision cardio-oncology. J Nucl Med. 2019;60:443-450.

28. Lewis JS, Windhorst AD, Zeglis BM, eds. Radiopharmaceutical Chemistry. New York, NY: Springer; 2019.

29. Sharma SK, Pourat J, Abdel-Atti D, et al. Noninvasive interrogation of DLL3 expression in metastatic small cell lung cancer. Cancer Res. 2017;77:39313941

30. Wang Y, Ayres KL, Goldman DA, et al. ${ }^{18} \mathrm{~F}$-fluoroestradiol PET/CT measurement of estrogen receptor suppression during a phase I trial of the novel estrogen receptor-targeted therapeutic GDC-0810: using an imaging biomarker to guide drug dosage in subsequent trials. Clin Cancer Res. 2017;23:3053-3060. 
31. Sanchez-Vega F, Hechtman JF, Castel P, et al. EGFR and MET amplifications determine response to HER2 inhibition in ERBB2-amplified esophagogastric cancer. Cancer Discov. 2019;9:199-209.

32. Surti S, Viswanath V, Daube-Witherspoom ME, Conti M, Casey ME, Karp JS. Benefit of improved performance with state-of-the art digital PET/CT for lesion detection in oncology. $J$ Nucl Med. March 20, 2020 [Epub ahead of print].

33. Cherry SR, Jones T, Karp JS, Qi J, Moses WW, Badawi RD. Total-body PET: maximizing sensitivity to create new opportunities for clinical research and patient care. J Nucl Med. 2018;59:3-12.

34. Mankoff DA, Pryma DA, Clark AS. Molecular imaging biomarkers for oncology clinical trials. J Nucl Med. 2014;55:525-528.

35. Lassmann M, Eberlein U. The relevance of dosimetry in precision medicine. J Nucl Med. 2018;59:1494-1499.

36. Wahl RL, Jacene H, Kasamon Y, Lodge MA. From RECIST to PERCIST: evolving considerations for PET response criteria in solid tumors. $\mathrm{J}$ Nucl Med. 2009;50(suppl 1):122S-150S.

37. Yu J, Nguyen HNY, Steenbergen W, Kim K. Recent development of technology and application of photoacoustic molecular imaging toward clinical translation. J Nucl Med. 2018;59:1202-1207.

38. Scott R. Artificial intelligence: its use in medical diagnosis. J Nucl Med. 1993;34:510514.

39. Links JM, Devous MD Sr. Detection and comparison of patterns in images. J Nucl Med. 1994;35:16-17.

40. Fujita H, Katafuchi T, Uehara T, Nishimura T. Application of artificial neural network to computer-aided diagnosis of coronary artery disease in myocardial SPECT bull's-eye images. J Nucl Med. 1992;33:272-276.
41. Kippenhan JS, Barker WW, Pascal S, Nagel J, Duara R. Evaluation of a neuralnetwork classifier for PET scans of normal and Alzheimer's disease subjects. J Nucl Med. 1992;33:1459-1467.

42. Vogel WV, Oyen WJ, Barentsz JO, Kaanders JH, Corstens FH. PET/CT: panacea, redundancy, or something in between? J Nucl Med. 2004;45(suppl 1):15S-24S.

43. Weber W, Young C, Abdel-Dayem HM, et al. Assessment of pulmonary lesions with ${ }^{18} \mathrm{~F}$-fluorodeoxyglucose positron imaging using coincidence mode gamma cameras. J Nucl Med. 1999;40:574-578.

44. Rowe SP, Gorin MA, Pomper MG. Imaging of prostate-specific membrane antigen with small-molecule PET radiotracers: from the bench to advanced clinical applications. Annu Rev Med. 2019;70:461-477.

45. Nunn AD. The cost of developing imaging agents for routine clinical use. Invest Radiol. 2006;41:206-212.

46. Seidlin SM, Marinelle LD, Oshri E. Radioactive iodine therapy; effect on functioning metastases of adenocarcinoma of the thyroid. J Am Med Assoc. 1946; 132:838-847.

47. Hertz S, Roberts A, Salter WT. Radioactive iodine as an indicator in thyroid physiology. IV. The metabolism of iodine in Graves' disease. J Clin Invest. 1942; 21:25-29.

48. Segall GM. Looking to the future: the ABNM in the next 10 years. $J$ Nucl Med. 2016;57(2):12N.

49. Czernin J. Roundtable on the future of nuclear medicine training [reply]. J Nucl Med. 2015;56:1969-1970.

50. Brettschneider J, Del Tredici K, Lee V, Trojanowski J. Spreading of pathology in neurodegenerative diseases: a focus on human studies. Nat Rev Neurosci. 2015;16:109_ 120. 